\title{
Increased gut microbiota diversity and abundance of Faecalibacterium prausnitzii and Akkermansia after fasting: a pilot study
}

\author{
Marlene Remely · Berit Hippe · Isabella Geretschlaeger · Sonja Stegmayer • Ingrid Hoefinger • \\ Alexander Haslberger
}

Received: 2 October 2014 / Accepted: 20 January 2015 / Published online: 13 March 2015

(C) Springer-Verlag Wien 2015

\begin{abstract}
Summary
Background An impaired gut microbiota has been reported as an important factor in the pathogenesis of obesity. Weight reduction has already been mentioned to improve gut microbial subpopulations involved in inflammatory processes, though other subpopulations still need further investigation. Thus, weight reduction in the context of a fasting program together with a probiotic intervention may improve the abundance and diversity of gut microbiota.

Methods In this pilot study, overweight people underwent a fasting program with laxative treatment for 1 week followed by a 6 week intervention with a probiotic formula. Gut microbiota were analyzed on the basis of $16 \mathrm{~s}$ rDNA with a quantitative real time polymerase chain reaction. Additionally, a food frequency questionnaire with questions about nutritional behavior, lifestyle, and physical activity was administered before and after the intervention.

Results We observed an increase in microbial diversity over the study period. No significant changes in abundance of total bacteria, or of Bacteroidetes, Prevotella, Clostridium cluster XIVa, or Clostridium cluster IV were found, although Faecalibacterium prausnitzii showed an increase over the study period. In addition, Akkermanisa and Bifidobacteria increased in abundance due to intervention. The inflammation-associated gut microbes Enterobacteria and Lactobacilli increased during the first
\end{abstract}

M. Remely $(\bowtie) \cdot$ B. Hippe $\cdot$ I. Geretschlaeger $\cdot$ S. Stegmayer $\cdot$

A. Haslberger

Institute of Nutritional Sciences, University Vienna,

Althanstraße 14,

1090 Vienna, Austria

e-mail: marlene.remely@univie.ac.at

I. Hoefinger

Essen \& Fasten by Ingrid Hoefinger,

Purkersdorf, Austria week and then declined by the end of the intervention. Two-thirds of the study participants harbored Archaea. No significant improvements of eating habits were reported, although physical activity improved due to the intervention.

Conclusions Our results show that caloric restriction affects gut microbiota by proliferating mucin-degrading microbial subpopulations. An additional intervention with a probiotic formula increased probiotic-administered gut microbial populations.

Keywords Akkermansia - Fasting · Laxative · Probiotic · Microbial diversity

$\begin{array}{ll}\text { Abbrevations } \\ \text { BMI } & \text { Body mass index } \\ \text { FFQ } & \text { Food frequency questionnaire } \\ \text { IBS } & \text { Irritable bowel syndrome } \\ \text { SCFAs } & \text { Short-chain fatty acids } \\ \text { SD } & \text { Standard deviation } \\ \text { T } & \text { Time point }\end{array}$

\section{Introduction}

Metabolic disorders (obesity, diabetes) are associated with changes in the microbiota as well as with hostmicrobiota interactions [1]. Pathogenic microorganisms are not always involved: members of the "healthy" microbiota are known to have an influence on disease state [2]. An altered intestinal composition or bacterial overgrowth can affect host health [3-5]. More specifically, obesity is associated with lower bacterial diversity, phylum and genus-level changes, and altered representation of bacterial genes and metabolic pathways involved in nutrient harvest. These differences involve members of the Bacteroidetes, Firmicutes, and Actinobacteria phyla of bacteria [6]. 
Thus it is expected that relief might be provided by fasting, through the adjustments needed to meet the macro- and micronutrient requirements of the body with limited food and to make use of the body's energy reserves without endangering health. This involves a limited period of abstinence from solid food and natural stimulants, with an intake of $2.5 \mathrm{~L} /$ day of calorie-free liquid (water, herbal tea) or vegetable broth (600-800 kcal/ day), which stimulates the excretory systems (liver, kidneys). The decreased use of energy for digestion and storage and metabolic energy-saving mechanisms, e.g., the shift from glucose to fat oxidation, allow sparing of essential proteins to maintain organs and cellular functions in the long-term. Though the biological necessity of fasting is long obsolete, its practice has developed in religious/spiritual contexts and for medical therapeutic purposes. One such school of fasting was developed following Dr. Otto Buchinger: a multidisciplinary and multimodal treatment comprising physiotherapy, nutrition, mind-body methods, and psychotherapy as well as physical activity.

Indications for fasting include metabolic syndrome and diseases, chronic inflammatory diseases, chronic cardiovascular diseases, chronic pain syndromes, atopic diseases, and psychosomatic disorders [7], but effects on gut microbiota might also demand consideration. However, cardiovascular, metabolic, and psychological changes must be taken into account and monitored: thus contraindications are cachexia, anorexia nervosa and other eating disorders, uncontrolled hyperthyroidism, advanced cerebrovascular insufficiency or dementia, advanced liver or kidney insufficiency, pregnancy, and nursing [7].

However, to date, there are hardly any studies about the influence of fasting on gut microbiota. One animal study showed that caloric restriction results in a change of the intestinal microbiota, namely an increase of Lactobacillus spp., which is thought to protect against invading pathogens and to reduce inflammatory cytokines. Streptococcacae, inducers of mild inflammation, are reported to be greatly reduced [8]. An additional treatment with probiotics might improve the impact of fasting: mucosal clearing of persistent gut microbiota facilitates the adherence of probiotic administered strains and improves the relief of gastrointestinal symptoms.

In this pilot study, we examined whether participation in a 1 week supervised fasting program in combination with a probiotic intervention modulates the relative abundance or composition of microorganisms in the gastrointestinal tract as measured by qPCR of the 16S rDNA.

\section{Materials and methods}

\section{Study participants and study design}

In accordance with the declaration of the Viennese Human Ethics committee (EK 14-092-VK_NZ), all study participants gave written consent for the use of data obtained from a food frequency questionnaire (FFQ) and stool samples. Thirteen individuals (aged 53.33 \pm 6.55 ages, BMI $28.10 \pm 3.50 \mathrm{~kg} / \mathrm{m}^{2}$ ) were recruited in cooperation with health trainer Mrs Ingrid Höfinger and Dr. Georg Wögerbauer (Monastery Pernegg, Austria). All participants joined a one-week fasting program prescribed by Dr. Buchinger in Pernegg Monastery, defined and supervised by Mrs. Höfinger. The fasting program specifies the following diet:

First day: breakfast: Pernegg muesli (prunes, dates, raisins, flaxseed, water); lunch: potatoes and vegetables; dinner: vegetable soup.

Second day: breakfast: herbal tea, Glauber's salt, lunch: fresh squeezed fruit and vegetable juice, dinner: fasting soup.

All other fasting days: breakfast: herbal tea, lunch: fresh squeezed fruit and vegetable juice, dinner: fasting soup.

On the second day of the fasting program, all participants received Glauber's salt (sodium sulfate) as a laxative to ensure full defecation. After the one-week fasting program, a probiotic intervention for 6 weeks started, using Progutic LactoVitamin BALANCE, which contains seven different DUOLAC ${ }^{\circ}$ bacterial strains per capsule: Lactobacillus plantarum, Streptococus thermophiles, Lactobacillus acidophilus, Lactobacillus rhamnosus, Bifidobacterium lactis, Bifidobacterium longum, and Bifidobacterium breve. In addition, a capsule contains fructooligosaccharides, $200 \mu \mathrm{g}$ folic acid, $2.50 \mu \mathrm{g}$ vitamin $\mathrm{B}_{12}$, and $55 \mu \mathrm{g}$ selenium (all amounts corresponding to $100 \%$ of daily demand) was also administered.

Fecal samples were collected at three time points: before fasting (T1), during fasting after sodium sulfate intake (T2), and after the 6 weeks of probiotic intervention (T3). Additionally, all participants were given a FFQ at the beginning (T1) and at the end of the intervention (T3). The FFQ reported the frequency of food consumption and portion size, as well as questions about lifestyle (i.e. smoking, alcohol consumption, physical activity), medically relevant influences (i.e. vitamin and other supplements), body mass index (BMI), and age to ensure comparable data.

\section{Stool sample processing and extraction}

Stool samples were collected and immediately stored at $-18^{\circ} \mathrm{C}$. Bacterial DNA was extracted from fecal samples using the QIAamp ${ }^{\circledR}$ DNA Stool mini kit (Qiagen, Germany) according to the manufacturer's protocol. In addition, samples were treated in FastPrep ${ }^{\text {TM }}$ Lysing Matrix E tubes (MP Biomedicals, USA) twice for $45 \mathrm{~s}$ in a beadbeater (Mini-Beadbeater 8 Bio-Spec Products, USA) with an intervening minute on ice. DNA concentration and quality of extraction was determined by Picol00 (Picodrop, UK) and agarose gel electrophoresis. 


\section{Real-time qPCR}

The abundance of bacteria and bacterial subgroups was measured by $16 \mathrm{~S}$ rDNA gene using TaqMan qPCR and SYBR Green qPCR in a Rotorgene 3000 (Corbett Life Science, Australia) with group-specific primers. Primers and probes were checked for specificity with the Probematch function of the Ribosomal Database Project release10 (http://rdp.cme.msu.edu/) [9]. The used primers, PCR reaction mixtures, and serial DNA dilutions of typical strains were prepared according to Remely et al. 2013 [10].

\section{Statistical analysis}

Differences between and within the groups were analyzed statistically with OriginPro version 8 (OriginLab, USA). All data were tested for normal distribution using the descriptive statistic normality test. For comparison of three unpaired groups the non-parametric Kruskal Wallis ANOVA was used, and for comparison of two nonparametric unpaired values, the non-parametric MannWhitney U-Test was used. For comparison between the time points, we used the Wilcoxon signed-rank test and the Friedman-Test. Correlations were determined using the Spearman correlation. In all tests $p$-values $<0.05$ were accepted as statistically significant.

\section{Results}

\section{Analyses of the retrospective FFQ}

Evaluation of the FFQ showed that only one out of six participants (16.67\%) consumed vegetables 5-10 times per week and two out of six (33.33\%) consumed fruits 5-10 times. In comparison, DACH guidelines recommend five portions of fruits and vegetables per day. According to DACH guidelines, meat and sausage are advised 2-3 times per week; $50 \%$ of participants complied with this recommendation, while $33.33 \%$ reported eating meat and sausages nearly every day. $83.33 \%$ consume fish 1-3 times per week and one participant 5-10 times per week. Only one-third of participants met the recommenda- tions for wheat and whole grain product consumption with daily intake, while $50 \%$ reported eating $<4$ portions per week. $33.33 \%$ consumed dairy products nearly every day, $50 \%$ of participants ate $<5$ portions per week, and only one out of six ate more than 15 portions per week. One third consumed sweets 1-3 times per week, another third 3-5 times and another third more than 10 times per week. In addition to the normal diet, $33.33 \%$ reported taking nutritional supplements (i.e. ascorbic acid, vitamin D, calcium, biotin). Additional questions about physical activity disclosed only one participant practicing daily movement, but $66.67 \%$ exercise regularly (1-3 times per week). Questions about stool behavior showed that $83.33 \%$ of participants documented no conscious problems with defecation.

The FFQ administered after probiotic intervention showed no significant differences in dairy product consumption, nor in the intake of meat, sausages, and fish compared to the first FFQ. There was also no observable increased intake of fruits and vegetables. Furthermore, the evaluation showed no increased or decreased intake of wheat, whole grain products, and sweets. There was a noticeable increase in daily movement in all participants, from 16.67 to $33.33 \%$, and regular physical activity improved from 66.67 to $83.33 \%$ of participants. After the fasting week and probiotic intervention, $100 \%$ documented no conscious problems with defecation. BMI did not change due to fasting (T1: 28.1 \pm 3.8 ; T3: 28.01 \pm 3.5 ).

\section{Compositional evaluation of gut microbiota}

In our pilot study, we detected some important differences in the gut bacterial composition before the fasting week and after probiotic intervention. Gut microbial diversity showed a significant increase due to fasting and probiotic intervention between the time points (T1-T3: $p=0.05$; T2-T3: $p=0.02$ ) with a mean at T1 of $15.5 \pm 3.1$ bands, T2 of $19.3 \pm 5.3$ and at T3 of $23.0 \pm 5.2$ bands (Fig. 1). No significant differences could be observed in total bacterial abundance between all three time points $(p=0.75)$. However, there was an increasing trend between $\mathrm{T} 1$ and $\mathrm{T} 2(p=0.47)$ and a decline from T2 to T3 $(p=0.81)$.

We observed no significant changes in the ratio of Firmicutes/Bacteroidetes between the three time points
Fig. 1 Diversity analysis. a PCR-DGGE fingerprinting of $16 S$ rDNA coding regions of dominant bacteria over time indicating a lower number of bands at $\mathrm{T} 1$ in comparison to T3. b Quantification of number of bands showing an increase of diversity between the time points. ( $T 1$ before fasting, 72 during fasting, T3 after 6 weeks of probiotic intervention)
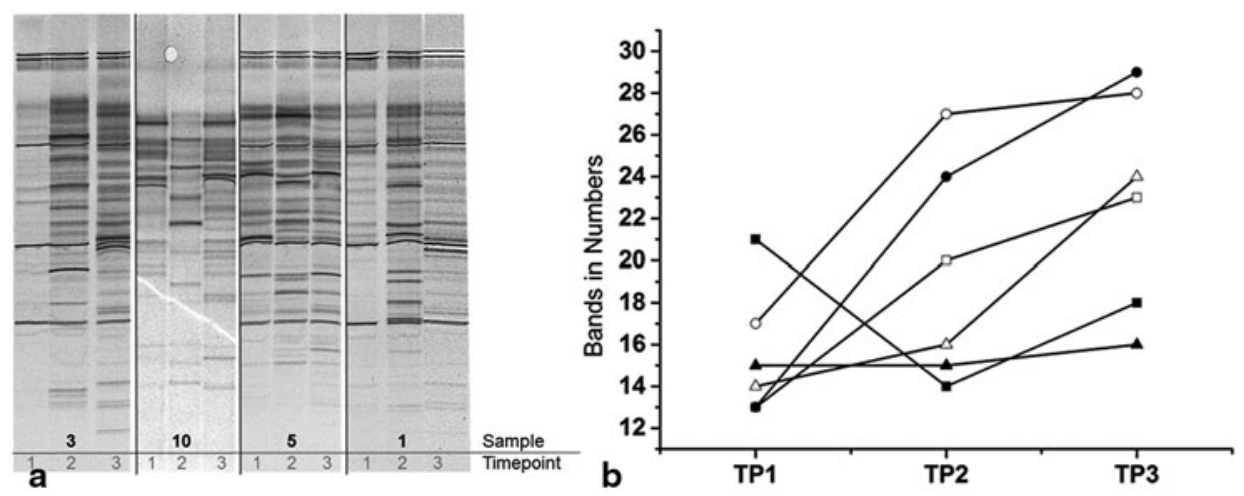
$(p=0.46)$. Furthermore, we detected no significant changes in the abundance of Clostridium Cluster IV ( $p=0.74)$, nor in the abundance of Clostridium Cluster XIVa $(p=0.71)$ between the three time points. Regarding Faecalibacterium prausnitzii, the dominant butyrate producer of Clostridium cluster $I V$, a significant increase was observed between the second and the third time point $(p=0.03)$, but no significant changes between $\mathrm{T} 1$ and $\mathrm{T} 2$ $(p=0.93)$. Lactobacilli show an increase from T1 to T2 $(p>0.01)$ and also from T1 to T3 $(p>0.01)$. There were no significant alterations in the abundance of Bacteroidetes $(p=0.59)$ and Prevotella $(p=0.81)$ over the study period. The abundance of Bifidobacteria increased significantly between T1 and T3 $(p=0.03)$ but remained constant between T1 and T2 ( $p=0.93)$. The quantity of Enterobacteria increased by tendency between T1 and T2 $(p=0.93)$ but declined between the last two time-points $(p=0.47)$. Akkermansia showed a significant increase between the time points (T1-T3: $\mathrm{p}=0.03$, T1-T2: $\mathrm{p}=0.47$, T2-T3: $\mathrm{p}=$ 0.47 ). Archaea were detected in $66.7 \%$ of the participants at all three time points.

\section{Discussion}

In the present pilot study, we have focused on patients suffering from obesity and intervened with a supervised fasting program according to Dr. Otto Buchinger, followed by an intervention with a probiotic formula to determine the feasibility of a further intervention study on gut microbiota in obesity.

After 1 week without solid food, subjects showed an increase in abundance of Lactobacilli, Enterobacteria, and Akkermansia. Other bacterial groups remained unaffected.

On the one hand, this effect may be attributed to caloric restriction; however, the supervised fasting program also included the intake of Glauber's salt, an osmotic laxative, on the second day of intervention, which might also affect the results. Laxatives are commonly used to facilitate defecation by increasing the intestinal passage, and decrease stool consistency and increase stool weight [11], and may result in a depletion of selective gut microbiota, especially transient species. Microbial organisms with a higher mucosal adherence, like mucin-degrading Akkermansia [12], remain nearly unaffected or may even proliferate due to a provided energy source [13]. Akkermansia are suggested to be greatly reduced in diverse inflammatory disorders (e.g., ulcerative colitis, Crohns' disease) [14] and thus are associated with a healthy microbial mucosa $[14,15]$. An induced osmotic diarrhea by polyethylene glycol 4000 (PEG) decreased the richness of phylotypes and showed a strong tendency to equalize the otherwise individualized microbiota on the mucosa. Bacteroidetes and Firmicutes significantly decrease [11] whereas Proteobacteria and Enterobacteria, proliferate in relative abundance of the mucosa specimens, a phenomenon also noted in several inflammatory and diarrheal gastrointestinal diseases $[11,16]$. Lactic acid bacteria also decreased on the mucosa, whereas Faecalibacterium showed a relative increase in stools, but a simultaneous decrease in the mucosa specimens [11].

Changes of the microbiota through dietary interventions are associated with alterations to the host's metabolic phenotype [6]. Metagenomic studies have demonstrated enrichment in gene coding for enzymes involved in the initial steps of breaking down indigestible dietary polysaccharides and in gene coding for enzymes [5]. Studies on obese mice show that the gut microbiome is enriched in genes encoding carbohydrate metabolism enzymes and has a greater capacity to extract energy from the diet and to generate short-chain fatty acids (SCFAs) $[4,5]$. In consequence, SCFAs play a role in the regulation of energy balance, inflammatory process, health, and obesity [17].

After the supervised fasting program, participants received an intervention with a probiotic formula. In particular, changes could be observed in the gut microbiota diversity and composition: Bifidobacteria and Akkermansia increased from the first time point to the third, and Faecalibacterium prausnitzii increased from the second time point to the third. Enterobacteria, which increased due to the fasting program, show a decrease to the third time point, whereas Lactobacilli still show an increase. Total bacterial abundance, Clostridium cluster IV, Clostridium cluster XIVa, Bacteroidetes, and Prevotella were unaffected, and Archaea are highly abundant at all three time points.

Studies on healthy individuals show that the intake of probiotic microorganisms leads in most cases to a transient colonization by the probiotic strains. Health effects are rarely studied or the outcomes are highly diverse [18]. However, some studies support the use of probiotics to alleviate pain and discomfort in patients suffering of lower gastrointestinal symptoms [19, 20]. Positive effects of probiotics have also been reported in the treatment or prevention of gastrointestinal inflammation-associated disorders such as traveler's diarrhea, antibiotics-associated diarrhea, or pouchitis [21]. However, the question remains which organisms are the most appropriate for different symptoms [20].

Archaea are highly abundant after the fasting program and together with the reduced nutritional intake, they support the thesis that Archaea encourage caloric intake in response to a hypocaloric diet, e.g. in anorexic patients [22, 23], but also due to fasting. Archaea improve bacterial fermentation efficiency through removal of $\mathrm{H}_{2}$ by a synothropic relationship [24, 25]. Thus, Archaea are expected to complicate weight loss or may even help prevent it [10].

\section{Conclusion}

In summary, our preliminary study shows that gut microbiota manipulation through fasting and laxatives may increase mucin-degrading subpopulations. An additional intervention with a probiotic formula was found to 
increase probiotic-administered gut microbial populations. These investigations are important to understand the gut microbiota within an individual through time and its response to dietary and environmental changes. Further research to produce clinical evidence to guide modification of the microbiota by predictive therapies and to prevent dysbiosis will be of great interest.

\section{Acknowledgement}

The work was funded by the Austrian Science Fund (FWF, AP2658721) and by the Abschlussstipendium 2014 of the University Vienna. The authors thank all participants without whom this study would not have been possible.

\section{Conflict of interest}

All authors declare that they have no actual or potential competing interests that might be perceived as influencing the results or interpretation of the reported study.

\section{References}

1. Hong SN, Rhee PL. Unraveling the ties between irritable bowel syndrome and intestinal microbiota. World J Gastroenterol. 2014;20(10):2470-81.

2. Ross RP, et al. Specific metabolite production by gut microbiota as a basis for probiotic function. Int Dairy J. 2009;20:269-76.

3. Owen N. An herbal therapeutic approach to food intolerance and immune dysfunction: an illustrative case history. J Herb Med. 2011;1:53-63.

4. Turnbaugh PJ, et al. A core gut microbiome in obese and lean twins. Nature. 2009;457(7228):480-4.

5. Turnbaugh PJ, et al. An obesity-associated gut microbiome with increased capacity for energy harvest. Nature. 2006;444(7122):1027-31.

6. Tremaroli V, Backhed F. Functional interactions between the gut microbiota and host metabolism. Nature. 2012;489(7415):242-9.

7. Toledo FWd, et al. Fasting therapy - an expert panel update of the 2002 consensus guidelines. Forsch Komplementmed. 2013;20:434-43.

8. Zhang C, et al. Structural modulation of gut microbiota in life-long calorie-restricted mice. Nat Commun. 2013;4:2163.

9. Cole JR, et al. Ribosomal database project: data and tools for high throughput rRNA analysis. Nucleic Acids Res. 2014;42(Database issue):D633-42.
10. Remely $\mathrm{M}$, et al. Abundance and diversity of microbiota in type 2 diabetes and obesity. Diabetes Metab Res Rev. 2013;4(4):3.

11. Gorkiewicz G, et al. Alterations in the colonic microbiota in response to osmotic diarrhea. PLoS One. 2013;8(2):e55817.

12. Derrien $\mathrm{M}$, et al. Akkermansia muciniphila gen. nov., sp. nov., a human intestinal mucin-degrading bacterium. Int J Syst Evol Microbiol. 2004;54(Pt 5):1469-76.

13. Sonnenburg JL, Angenent LT, Gordon JI. Getting a grip on things: how do communities of bacterial symbionts become established in our intestine? Nat Immunol. 2004;5(6):569-73.

14. Png CW, et al. Mucolytic bacteria with increased prevalence in IBD mucosa augment in vitro utilization of mucin by other bacteria. Am J Gastroenterol. 2010;105(11):2420-8.

15. Belzer C, de Vos WM. Microbes inside-from diversity to function: the case of Akkermansia. ISME J. 2012;6(8):1449-58.

16. Allen-Vercoe E, Jobin C. Fusobacterium and Enterobacteriaceae: important players for CRC? Immunol Lett. 2014;162:54-61.

17. Delzenne NM, Cani PD. Interaction between obesity and the gut microbiota: relevance in nutrition. Annu Rev Nutr. 2011;31:15-31.

18. Gerritsen J, et al. Intestinal microbiota in human health and disease: the impact of probiotics. Genes Nutr. 2011;6(3):209-40.

19. Hungin AP, et al. Systematic review: probiotics in the management of lower gastrointestinal symptoms in clinical practice - an evidence-based international guide. Aliment Pharmacol Ther. 2013;38(8):864-86.

20. Simren $M$, et al. Intestinal microbiota in functional bowel disorders: a Rome foundation report. Gut. 2012;62(1):159-76.

21. Quigley EMM, Flourie B. Probiotics and irritable bowel syndrome: a rationale for their use and an assessment of the evidence to date. Neurogastroenterol Motil. 2007;19:166-72.

22. Arumugam $M$, et al. Enterotypes of the human gut microbiome. Nature. 2011;473(7346):174-80.

23. Dridi B, Raoult D, Drancourt M. Archaea as emerging organisms in complex human microbiomes. Anaerobe. 2011;17(2):56-63.

24. DiBaise JK, et al. Gut microbiota and its possible relationship with obesity. Mayo Clin Proc. 2008;83(4):460-9.

25. Gill SR, et al. Metagenomic analysis of the human distal gut microbiome. Science. 2006;312(5778):1355-9. 